\title{
Analysis of $\mathrm{PKC}-\zeta$ protein levels in normal and malignant breast tissue subtypes
}

\author{
TRACESS SMALLEY, S. M. ANISUL ISLAM, CHRISTOPHER APOSTOLATOS, \\ ANDRÉ APOSTOLATOS and MILDRED ACEVEDO-DUNCAN
}

Department of Chemistry, The University of South Florida, Tampa, FL 33620, USA

Received February 16, 2018; Accepted November 9, 2018

DOI: $10.3892 / \mathrm{ol} .2018 .9792$

\begin{abstract}
It is estimated that breast cancer will be the second leading cause of cancer-associated mortality in women in 2018. Previous research has demonstrated that the atypical protein kinase $\mathrm{C}-\zeta(\mathrm{PKC}-\zeta)$ is a component of numerous dysregulated pathways in breast cancer, including cellular proliferation, survival, and cell cycle upregulation. The present study investigated the PKC- $\zeta$ protein in breast tissue to evaluate its potential as a biomarker for breast cancer invasion, and demonstrated that an overexpression of $\mathrm{PKC}-\zeta$ protein can be indicative of carcinogenesis. The present study analyzed the expression of PKC- $\zeta$ in individuals with no tumor complications and malignant female human breast tissue samples (lobular carcinoma in situ, invasive lobular carcinoma, ductal carcinoma in situ and invasive ductal carcinoma) with the use of western blot analysis, immunohistochemistry and statistical analysis (83 samples). The present study also evaluated the invasive behavior of MDA-MB-231 breast cancer cells following the knockdown of PKC- $\zeta$ with a Transwell invasion assay and an immunofluorescent probe for filamentous actin (F-actin) organization. The data demonstrated that $\mathrm{PKC}-\zeta$ expression was identified to be higher in invading tissues when compared with non-invading tissues. The results also suggest that PKC- $\zeta$ is more abundant in ductal tissues when compared with lobular tissues. In addition, the protein studies also suggest that PKC- $\zeta$ is a component for invasive behavior through the Ras-related C3 botulinum toxin substrate 1 (Rac1) and Ras homolog gene family member A (RhoA) pathway, and $\mathrm{PKC}-\zeta$ is required for the F-actin reorganization in invasive cells. Therefore, $\mathrm{PKC}-\zeta$
\end{abstract}

Correspondence to: Dr Mildred Acevedo-Duncan, Department of Chemistry, The University of South Florida, 4202 East Fowler Avenue, Tampa, FL 33620, USA

E-mail: macevedo@usf.edu

Abbreviations: $\mathrm{PKC}-\zeta$, protein kinase $\mathrm{C} \zeta$; PRKCZ, gene name for protein kinase $\mathrm{C} \zeta$; $\mathrm{CDH} 1$, gene name for E-cadherin; LCIS, lobular carcinoma in situ; ILC, invasive lobular carcinoma; DCIS, ductal carcinoma in situ; IDC, invasive ductal carcinoma

Key words: breast cancer, biomarkers, protein kinase $\mathrm{C} \zeta$ should be considered to be a biomarker in the development of breast cancer as well as an indicator of invading tumor cells.

\section{Introduction}

In the current research, cancer statistics show that invasive breast cancer is projected to have 234,190 new cases and deaths, for which 231,840 will be women (1). Approximately 40,290 women will die from invasive breast cancer in 2018 (2). Although the percentage of mortalities has decreased over the last few years, breast cancer still ranks as the second leading cause of cancer death in women; treatments usually entail invasive surgeries, including breast-conserving surgeries and mastectomies (1). From a clinical standpoint, invasive ductal carcinoma (IDC) is the most common form of breast cancer, affecting $50-75 \%$ of breast cancer diagnoses (2). Breast cancer is a heterogeneous disease, and research has shown that the top mutated genes in breast carcinomas and carcinomas in situ are Phosphatidylinositol-4,5-Bisphosphate 3-Kinase Catalytic Subunita (PIK3CA), Tumor Protein P53 (TP53), and E-cadherin (CDH1) (3). However, this investigation shows a link between protein kinase $\mathrm{C}-\zeta$ (PKC- $\zeta)$ protein overexpression and breast cancer development, particularly in invasive behavior. For this reason, breast cancer biomarkers are of interest, as they may help to predict breast cancer incidents and contribute to better therapeutic regimens.

The complete understanding of histological and pathological features of the lobular and ductal carcinomas are far from full elucidation. In an investigation by Ruibal et al, the authors concluded that, in the absence of the axillary node, lobular carcinomas had a higher concentration of breast cancer estrogen-inducible protein $(\mathrm{pS} 2)$ than ductal carcinomas (4). Lobular carcinomas were also shown to have a higher frequency of diploidy, which suggests that lobular carcinomas are less aggressive and grow slower (4).

An overexpression of $\mathrm{PKC}-\zeta$ protein promotes carcinogenesis by stimulating cancer cell proliferation through pathways such as the Nuclear factor- $\kappa \mathrm{B}(\mathrm{NF}-\kappa \mathrm{B})$, which plays an essential role in cancer initiation and progression (5). Previous studies suggest that $\mathrm{PKC}-\zeta$ is a regulatory factor for the nuclear translocation of $\mathrm{NF}-\kappa \mathrm{B}$ that in turn represses E-cadherin (6,7). Although the link between the loss of E-cadherin and cancer prognosis remains ambiguous, recent findings showed that E-cadherin possesses a vital tumor suppressive role (8). Moreover, some researchers 
have paralleled PKC- $\zeta$ to the phosphorylation of the Inhibitor of $\kappa \mathrm{B}$ kinase (IKK) complex, which in turn phosphorylates the Inhibitor of $\kappa \mathrm{B}(\mathrm{I} \kappa \mathrm{B})$ and triggers I $\mathrm{B}$ degradation $(7,9)$. The degradation of $\mathrm{I} \kappa \mathrm{B}$ releases $\mathrm{NF}-\kappa \mathrm{B}$, allowing its translocation from the cytosol into the nucleus, where it functions as a transcription factor (9). The transcription factor applies explicitly to targets such as apoptosis regulators and stress response genes. Furthermore, studies show that NF- $\kappa \mathrm{B}$ also plays a role in epithelial to mesenchymal transition, a crucial carcinogenic event (7).

PKC $-\zeta$ has also been linked to metastatic behaviors of cancer cells. In a study by Islam et al, the Ras-related C3 botulinum toxin substrate 1 (Rac1)/Pak1/ $\beta$-Catenin signaling cascade in colorectal cancer cell lines was evaluated after the inhibition of PKC- $\zeta$ (10). The knockdown of PKC- $\zeta$ decreased the nuclear translocation of $\beta$-Catenin which ultimately leads to reduced colorectal cell proliferation and metastasis (10). These data were further supported by another investigation performed by Wu et al, which determined that inhibition of PKC- $\zeta$ in breast cancer cell lines decreased adhesion and actin polymerization (11). These studies advocate the theory that PKC- $\zeta$ as a critical component of the invasive behaviors of cancer cells.

Although PKC- $\zeta$ has been studied in invasive breast cancers (most commonly ductal), there have been no examinations of PKC- $\zeta$ expression in other types of breast cancer (such as carcinomas in situ). An investigation by Lin et al confirmed that atypical PKC isoforms were elevated in breast cancer tissues (IDC, specifically) when compared to adjacent healthy breast tissue (12). Additionally, Schöndorf et al determined that antineoplastic agents affect the activation of PKC in IDC breast cancer tumors (13). In this study, our focus was to further investigate the PKC- $\zeta$ expression profile in the four histological subtypes of breast cancer such as lobular carcinoma in situ (LCIS), invasive lobular carcinoma (ILC), ductal carcinoma in situ (DCIS) and IDC. We also evaluated the difference in the PKC- $\zeta$ expression among healthy, invasive and non-invasive tissues. Moreover, the invasive characteristics of MDA-MB-231 breast cancer cells were examined upon the inhibition of $\mathrm{PKC}-\zeta$. We found that $\mathrm{PKC}-\zeta$ is overexpressed in IDC and ILC tissue specimens compared to other subtypes. In addition, the inhibition of PKC- $\zeta$ decreased the invasion of MDA-MB-231 breast cancer cells.

\section{Materials and methods}

Specimen collection and tissue fractionation. The NCI-supported Cooperative Human Tissue Network (CHTN; Birmingham, AL, USA) collected and provided the breast tissue samples (normal, LCIS, ILC, DCIS, and IDC). The specimens were collected and snap-frozen in liquid nitrogen or placed in dry ice and stored in a liquid nitrogen vapor phase freezer $\left(-196^{\circ} \mathrm{C}\right)$, where the tissues stayed until shipment. The tissues were shipped in dry ice. Formalin-fixed paraffin-embedded tissues (FFPE) were also provided for immunohistochemistry staining. Tissues were selected based on their histological features (normal, LCIS, ILC, DCIS, and IDC). The mean age of patient samples collected was 51 years and the collection period of the samples was 2001-2015. Normal tissues were selected from breast reduction patients with no previous diagnosis of cancer or the area adjacent to a patient's malignant tumors. Patients with DCIS and LCIS were selected based on the lack of invasive tissue adjacent to the extraction site; some tissues were taken from patients with invasive tissues in the opposite breast.

The tissue specimens were then resuspended and sonicated for $3 \times 5 \mathrm{sec}$ cycles on ice in $1 \mathrm{ml}$ of homogenization buffer (Pierce ${ }^{\circledR}$ Immuno Precipitation Lysis Buffer, $25 \mathrm{mM}$ Tris- $\mathrm{HCl}$ $\mathrm{pH} 7.4,150 \mathrm{mM} \mathrm{NaCl}, 1 \mathrm{mMEDTA}, 1 \% \mathrm{NP}-40$ and 5\% glycerol; Thermo Fisher Scientific, Inc., Waltham, MA, USA), followed by, centrifugation at $12,879 \mathrm{x}$ g for $15 \mathrm{~min}$ at $4^{\circ} \mathrm{C}$ to obtain cell extracts. Subsequently, $250 \mu 1$ of albumin removal resin (Pierce $^{\mathrm{TM}}$ Albumin Depletion kit; Thermo Fisher Scientific, Inc.) was added to the lysate and left at $4^{\circ} \mathrm{C}$ overnight. The samples were further centrifuged at $12,879 \mathrm{x} g$ for $15 \mathrm{~min}$ at $4^{\circ} \mathrm{C}$, the supernatant was subsequently collected, and protein content was measured according to Pierce ${ }^{\circledR} 660 \mathrm{~nm}$ Protein Assay reagent protocol (Thermo Fisher Scientific, Inc.) and NanoDrop 2000 (Thermo Fisher Scientific, Inc., Wilmington, DE, USA).

Cell culture. The metastatic breast cancer cell line, MDA-MB-231 was obtained from American Type Tissue Culture Collection (ATCC; Manassas, VA, USA). The MDA-MB-231 cells were sub-cultured and maintained in T75 flasks containing Dulbecco's modified Eagle's media (DMEM; Gibco; Thermo Fisher Scientific, Inc., Waltham, MA, USA), supplemented with $10 \%$ fetal bovine serum (FBS) and $1 \%$ antibiotics (penicillin $10 \mathrm{U} / \mathrm{ml}$ and streptomycin $10 \mathrm{mg} / \mathrm{ml}$ ). Cells were incubated at $37^{\circ} \mathrm{C}$ and $5 \% \mathrm{CO}_{2}$. Cells were used at $70-80 \%$ confluency for the experiments.

Western blot analysis. Like tissue lysates, MDA-MB-231 cell extracts were also prepared after the addition of cell lysis buffer, sonication, and centrifugation (at 12,879 $\mathrm{x} g$ for $15 \mathrm{~min}$ at $4^{\circ} \mathrm{C}$ ). For western blot analysis, an equal amount (20-30 $\mu \mathrm{g}$ ) of protein from tissue and cell lysates were loaded in $10 \%$ polyacrylamide gels and separated by using sodium dodecyl sulfate-polyacrylamide gel electrophoresis (SDS-PAGE). The proteins were then transblotted onto a $0.4 \mu \mathrm{M}$ nitrocellulose membrane. Subsequently, the membranes were incubated with $5 \%$ milk blocking solution, followed by, primary solution of anti-PKC- $\zeta$ (SC-17781; 1:1,000; Santa Cruz Biotechnology, Dallas, TX, USA; and 9372s; 1:1,000; Cell Signaling Technology, Danvers, MA, USA), anti-E-cadherin (701134; 1:1,000; Invitrogen; Thermo Fisher Scientific, Inc.), anti-Ras homolog gene family member A (RhoA; ab54835, 1:4,000; Abcam, Cambridge, UK) and anti-Rac1 (4651s; 1:1,000; Cell Signaling Technology) in 5\% bovine serum albumin (BSA). Finally, the membranes were incubated with secondary antibodies. All the secondary antibodies (anti-rabbit and anti-mouse) were obtained from Bio-Rad Laboratories, Hercules, CA, USA (cat. no. 170-6515 and cat. no. 170-6516; 1:2,000). The immunoreactive bands were then visualized by chemiluminescence reaction, according to the manufacturer's instructions (SuperSignal ${ }^{\mathrm{TM}}$ West Pico PLUS Chemiluminescent Substrate; Thermo Fisher Scientific, Inc.). A monoclonal antibody to $\beta$-actin (SC-1616; Santa Cruz Biotechnology) was used as a loading control. 
$\mathbf{A}$
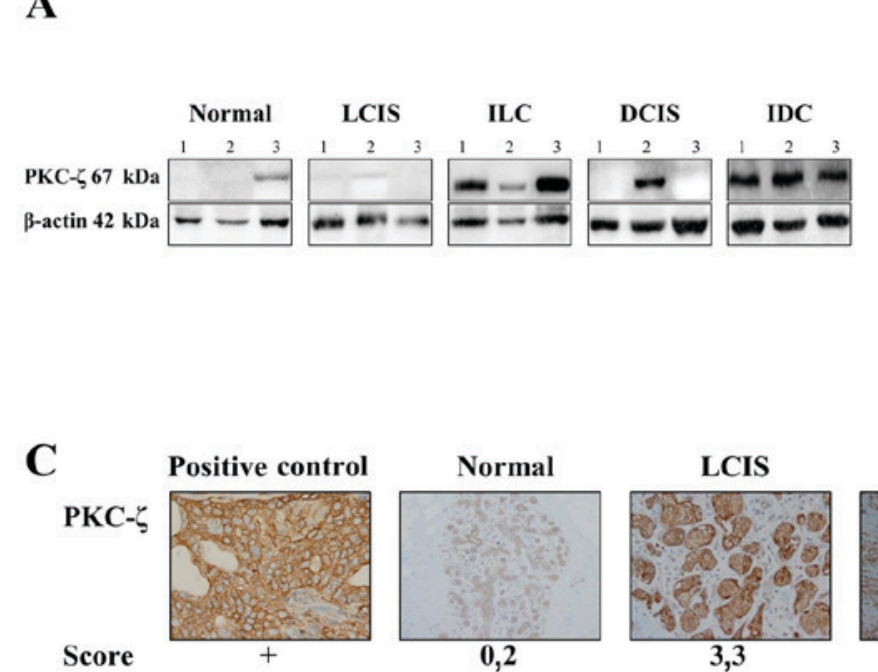

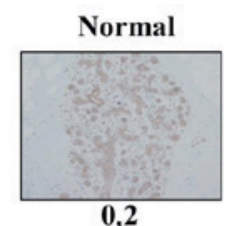

0,2

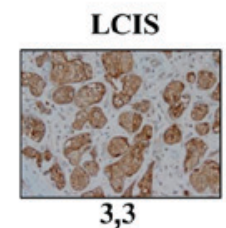

3,3
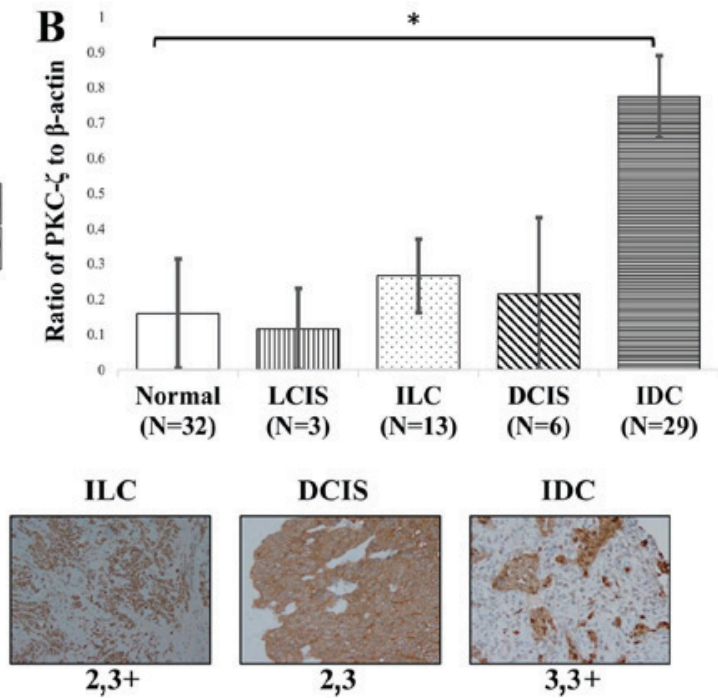

Figure 1. Western blot and immunohistochemistry analysis of PKC- $\zeta$ expression in normal and malignant tissues. Malignant tissues were evaluated for the expression of PKC- $\zeta$. (A) We ran 20-30 $\mu \mathrm{g}$ of tissue lysate and probed with a PKC- $\zeta$ antibody. (B) The ratios of PKC- $\zeta$ to $\beta$-actin in healthy tissue and the four subtypes of malignant breast tissue were derived using the densitometry. The white bar (healthy) represents the control and the error bars represent the standard error. The standard deviation, a Student's t-test $(\mathrm{P}<0.05)$ and a one-way ANOVA was used to evaluate the western blot data as well with the post-hoc Tukey's HSD test $(\mathrm{P}<0.01)$, Scheffé multiple comparison $(\mathrm{P}<0.05)$, Bonferroni $(\mathrm{P}<0.01)$ and Holm $(\mathrm{P}<0.01)$. Statistical tests showed no meaningful relationship between the healthy and LCIS, or the ILC and DCIS. *, statistically relevant relationship between normal and IDC breast tissue. (C) FFPE samples were probed with PKC- $\zeta$ and stained for immunohistochemistry. The scoring was based on the abundance or lack thereof PKC- $\zeta$ expression: Score: 0 , no expression; 1 , weak expression; 2 , moderate expression; 3, abundant expression; 3 plus (+), strong expression. Image original magnification, x20. LCIS, lobular carcinoma in situ; ILC, invasive lobular carcinoma; IDC, invasive ductal carcinoma; DCIS, ductal carcinoma in situ; PKC- $\zeta$, protein kinase C- $\zeta$; FFPE, formalin-fixed paraffin-embedded tissues.

Densitometry. The densitometry was performed using Image $\mathrm{J}$ (National Institutes of Health, Bethesda, MD, USA) software by the subtraction of background noise from the density of each band to derive the corrected intensity. All samples were normalized based on the intensity of $\beta$-actin bands on each blot.

Immunohistochemistry. FFPE tissues received from CHTN were sent to the Tissue Core, Moffitt Cancer Center (Tampa, FL, USA). Two different patient tissues were selected for each subtype (normal, LCIS, ILC, DCIS, and IDC) based on the criteria mentioned previously, with a total $N=10$. Briefly, tissues were stained with haemotoxylin and eosin (H\&E), and pathology quality control (PQC) were performed by a Tissue Core pathologist to confirm breast tissue subtype and diagnosis. The slides were prepared and stained with antibodies for PKC- $\zeta$ (ab59364; 1:1,000; rabbit polyclonal; Abcam). A uterine carcinoma specimen was selected as a positive control based on antibody data sheet and the Human Protein Atlas recommendations. Slides were stained using a Ventana Discovery XT automated system (Ventana Medical Systems, Tucson, AZ, USA) as per the manufacturer's protocol with proprietary reagents. Briefly, slides were deparaffinized on the automated system with EZ Prep solution (Ventana Medical Systems). The heat-induced antigen retrieval method ( $\mathrm{CC} 1$ standard) was used with the PKC- $\zeta$ primary rabbit antibody (Ventana Medical Systems). This antibody that reacts to the human isoform of PKC- $\zeta$ was used at a 1:1,000 concentration in Dako antibody diluent (Dako; Agilent Technologies, Inc., Santa Clara, CA, USA) and incubated for $32 \mathrm{~min}$. The Ventana OmniMap Anti-Rabbit Secondary Antibody (Ventana Medical Systems) was used for $16 \mathrm{~min}$. The detection system used was the Ventana ChromoMap kit and slides were then counterstained with haematoxylin. Slides were subsequently dehydrated and cover-slipped as per normal laboratory protocol. The Moffitt
Cancer Center Tissue Core pathologist selected the optimal condition, titration, and incubation time to be used on the control and the breast selected slides. Subsequently, the pathologist evaluated the slides using the combinative semi quantitative scores (score, 0-3) (14). Images were taken on a light microscope Olympus BX51 (Olympus Corp., Tokyo, Japan).

Knockdown of PKC- $\zeta$ for invasion pathway analysis. Human breast cancer cells MDA-MB-231 were grown in $100 \mathrm{~mm}$ plates and transfected with $20 \mathrm{nM}$ of scrambled RNA and siPRKCZ (5'-GCAUGAUGACGAGGAUAUUGACUGG-3', SR303747A; Origene, Rockville, MD, USA) for 48 h. Cells were lysed as previously described and the lysates were run on western blots.

Cell invasion assay by crystal violet staining of invaded cells. Cells were serum starved for $24 \mathrm{~h}$, followed by detachment and plating into the upper chamber of a 96-well $8 \mu \mathrm{m}$ Transwell permeable support, coated with $0.5 \mathrm{X}$ basement membrane extract (BME; both Corning Inc., Corning, NY, USA) for the evaluation of invasion. Serum (10\%) containing media was loaded into the receiver plate (lower chamber) as a chemoattractant. MDA-MB-231 cells at the upper chamber were transfected with $20 \mathrm{nM}$ siPRKCZ for $24 \mathrm{~h}$. Four experimental treatment groups for the cells were performed: Control (non-treated), Si-Tran (transfection reagent), scrambled siRNA (random RNA) and siRNA for PRKCZ (for knockdown of $\mathrm{PKC}-\zeta$ protein expression). The cells were treated with the transfection reagent (Si-Tran) and universal scrambled RNA to establish the effect of targeted small interfering RNA (siPRKCZ) only. The invasive cells that passed into the lower chamber were then fixed with $4 \%$ paraformaldehyde, stained with $2 \%$ crystal violet in $2 \%$ ethanol, washed with distilled 
Table I. The selection of breast specimens with pathological characteristics.

\begin{tabular}{|c|c|c|c|c|c|c|c|}
\hline $\mathrm{LN}$ & Grade/stage & ER & PR & Histology & Race & PKC- $\zeta$ expression & Number of patient samples \\
\hline NA & NA & NA & NA & Normal & Black & No & 9 \\
\hline NA & NA & NA & NA & Normal & NA & No & 4 \\
\hline NA & NA & NA & NA & Normal & White & No & 15 \\
\hline NA & NA & NA & NA & Normal & White & Yes & 4 \\
\hline NA & NA & NA & NA & LCIS & Black & No & 1 \\
\hline+ & II/III & - & - & LCIS & White & No & 1 \\
\hline+ & II/III & + & + & LCIS & White & Yes & 1 \\
\hline NA & NA & NA & NA & ILC & Black & No & 1 \\
\hline- & II/III & NA & NA & ILC & NA & No & 1 \\
\hline+ & III/III & + & + & ILC & NA & No & 1 \\
\hline NA & NA & NA & NA & ILC & NA & No & 1 \\
\hline- & $\mathrm{I} / \mathrm{III}$ & + & + & ILC & White & No & 1 \\
\hline- & NA & NA & NA & ILC & White & No & 1 \\
\hline+ & II/III & + & + & ILC & White & No & 1 \\
\hline NA & NA & NA & NA & ILC & White & No & 1 \\
\hline+ & II/III & + & + & ILC & Black & Yes & 1 \\
\hline+ & III/III & + & + & ILC & NA & Yes & 1 \\
\hline NA & NA & NA & NA & ILC & NA & Yes & 1 \\
\hline- & NA & + & + & ILC & White & Yes & 1 \\
\hline NA & $\mathrm{I} / \mathrm{III}$ & + & - & ILC & White & Yes & 1 \\
\hline- & III/III & - & + & IDC & Black & No & 1 \\
\hline+ & NA & - & + & IDC & Black & No & 1 \\
\hline NA & NA & NA & NA & IDC & NA & No & 1 \\
\hline- & $\mathrm{I} / \mathrm{III}$ & + & + & IDC & White & No & 1 \\
\hline NA & III/III & NA & NA & IDC & White & No & 1 \\
\hline NA & NA & + & - & IDC & White & No & 1 \\
\hline NA & NA & + & + & IDC & Black & Yes & 1 \\
\hline- & II/III & NA & NA & IDC & NA & Yes & 1 \\
\hline+ & III/III & NA & NA & IDC & NA & Yes & 1 \\
\hline+ & III/III & NA & NA & IDC & NA & Yes & 1 \\
\hline NA & NA & + & - & IDC & NA & Yes & 1 \\
\hline NA & NA & NA & NA & IDC & NA & Yes & 2 \\
\hline- & III/III & - & - & IDC & White & Yes & 2 \\
\hline+ & II/I & + & + & IDC & White & Yes & 1 \\
\hline+ & NA & - & - & IDC & White & Yes & 2 \\
\hline+ & NA & - & NA & IDC & White & Yes & 1 \\
\hline+ & NA & + & + & IDC & White & Yes & 1 \\
\hline+ & NA & NA & NA & IDC & White & Yes & 2 \\
\hline NA & $\mathrm{I} / \mathrm{III}$ & NA & NA & IDC & White & Yes & 1 \\
\hline NA & NA & - & - & IDC & White & Yes & 1 \\
\hline NA & NA & + & - & IDC & White & Yes & 2 \\
\hline NA & NA & NA & NA & IDC & White & Yes & 3 \\
\hline NA & NA & NA & NA & DCIS & Black & No & 1 \\
\hline NA & NA & NA & NA & DCIS & NA & No & 4 \\
\hline- & NA & NA & NA & DCIS & Black & Yes & 1 \\
\hline
\end{tabular}

Tissue specimen were selected as described in material and methods and further sorted by the presence of PKC- $\zeta$ protein. LN, lymph node; ER, estrogen receptor; PR, progesterone receptor; $\mathrm{PKC}-\zeta$, protein kinase $\mathrm{C}-\zeta$; LCIS, lobular carcinoma in situ; ILC, invasive lobular carcinoma; IDC, invasive ductal carcinoma; DCIS, ductal carcinoma in situ; NA, not available.

water and photographs were captured after drying using a light microscope Motic AE31E.
Phalloidin staining of filamentous actin (F-actin). Human breast cancer cells MDA-MB-231 ( $1 \times 10^{4}$ cells) were grown 
Table II. The status of $\mathrm{PKC}-\zeta$ in malignant and healthy breast tissue.

\begin{tabular}{lccc}
\hline Tissue type & $\begin{array}{c}\text { Not } \\
\text { present }\end{array}$ & $\begin{array}{c}\text { Weakly } \\
\text { present }\end{array}$ & $\begin{array}{c}\text { Positively } \\
\text { present }\end{array}$ \\
\hline Normal tissue & 29 & 1 & 2 \\
LCIS & 2 & 1 & 0 \\
ILC & 8 & 4 & 1 \\
DCIS & 5 & 0 & 1 \\
IDC & 6 & 5 & 18 \\
\hline
\end{tabular}

Healthy breast tissue was obtained from breast reductions or the area adjacent to a patient's malignant tumors. Malignant tumors were either LCIS, ILC, DCIS, or IDC. The expression of PKC- $\zeta$ was evaluated as not present (ratio 0 ), weakly present (ratio $<0.01$ ) and positively present (ratio >0.01). LCIS, Lobular carcinoma in situ; ILC, invasive lobular carcinoma; DCIS, ductal carcinoma in situ; IDC, invasive ductal carcinoma; $\mathrm{PKC}-\zeta$, protein kinase $\mathrm{C}-\zeta$.

in 2-well chamber slides, followed by transfection with $20 \mathrm{nM}$ universal scrambled RNA and siPRKCZ for $24 \mathrm{~h}$. In addition, cells were also evaluated with the transfection reagent and without any treatment to establish the targeted effect of PKC- $\zeta$ knockdown. Fixation was performed with 4\% paraformaldehyde. F-actin was subsequently stained with 1X Phalloidin-iFluor 594 (Abcam) in 1\% BSA-phosphate buffered saline (PBS) solution for an hour at room temperature. Cells were washed, counterstained with the nuclear stain 4',6-diamidino-2-phenylindole (DAPI; Invitrogen; Thermo Fisher Scientific, Inc.) and examined under Nikon MICROPHOT-FX fluorescence microscope (Ex/Em=590/618). Photographs were captured using ProgRes ${ }^{\circledR}$ Capture 2.9.0.1.

Statistical analysis. The statistical significance of the western blot analysis data was evaluated by a Student's t-test (normal $N=32$; LCIS $N=3$; ILC $N=13$; DCIS $N=6$; IDC $N=29$; overall $N=83$ at $\mathrm{P}<0.05$; standard error represented) and the linear regression test ( $N=20 ; \mathrm{R}^{2}$ value) with GraphPad software (15). A one-way ANOVA was also used to evaluate the western blot analysis data as well with the post-hoc Tukey's HSD test $(\mathrm{P}<0.01)$, Scheffé multiple comparison $(\mathrm{P}<0.05)$, Bonferroni $(\mathrm{P}<0.01)$ and Holm $(\mathrm{P}<0.01)$. The contingency table Chi-squared statistical analysis (normal $N=32$; LCIS $N=3$; ILC $N=13$; DCIS $N=6$; IDC $N=29$; overall $N=83$ at $\mathrm{P}<0.00001$ ) for the expression of PKC- $\zeta$ and breast subtype was performed using the Chi-Squared Test Calculator from Social Science Statistics (16). Clinical parameters such as estrogen receptor expression and Scarff-Bloom-Richardson grade (presented in the pathology reports) were also investigated with this statistical software ( $N=25$ and $N=22$, respectively). A one-way ANOVA was used to analyze the number of cells invaded after crystal violet staining $(\mathrm{P}<0.05)$.

\section{Results}

PKC- $\zeta$ is overexpressed in malignant breast tissues. PKC- $\zeta$ protein expression was measured in breast samples with no tumor complication and malignant breast tissue samples by western blot analysis. Our investigation showed a correlation between the overexpression of PKC- $\zeta$ and malignant breast cancer tissue (Fig. 1A and B; Table III). It was challenging to obtain DCIS $(N=6)$ and LCIS $(N=3)$ since these tissue types have lower occurrence rates; hence the results reflect a comparison of the two main subtypes: IDC, $N=29,(50-75 \%$ diagnoses) and ILC, $N=13$ (10-15\% diagnoses). The westerns represent the majority of samples. Normal tissue only had 3 of 32 samples with expression of PKC- $\zeta$ and DCIS only had 1 of 6 samples with PKC- $\zeta$ present (Table II). Among the IDC subtypes of malignant breast tissue, $>74 \%$ of tissue samples overexpressed PKC- $\zeta$ protein. Less than $5 \%$ of healthy breast tissue samples exhibited $\mathrm{PKC}-\zeta$ protein expression. Although a paired Student's t-test $(\mathrm{P}<0.05)$ and one-way ANOVA $(\mathrm{P}<0.01)$ showed no significant correlation between the overexpression of PKC- $\zeta$ protein in malignant tissue (all four subtypes) and healthy tissue, it did demonstrate a significant correlation between healthy breast tissue and IDC (Tukey HSD P-value 0.0056404, $\mathrm{P}<0.01$; Scheffé $\mathrm{P}$-value 0.0121289, $\mathrm{P}<0.05$; Bonferroni and Holm P-value 0.0063104, P<0.01) (Fig. 1B). According to the significance of the contingency table (Table III), there is an established association between the protein expression of $\mathrm{PKC}-\zeta$ and sample type $(\mathrm{P}<0.00001)$. However, the PKC $-\zeta$ expression could not be statistically linked to the presence of ER and nuclear grade (Table IV; Fig 3C). These data suggest that it is unlikely that healthy breast tissue samples overexpress PKC- $\zeta$ protein. Instead, PKC- $\zeta$ is overexpressed in malignant breast tissue samples.

$P K C-\zeta$ protein levels are higher in invading breast cancer subtypes. To compare the expression of $\mathrm{PKC}-\zeta$ in non-invading tissues and invading tissues, western blot and immunohistochemistry were performed. Western blot analysis data showed that $\mathrm{PKC}-\zeta$ protein expression was higher in IDC when compared to the ILC, LCIS and the DCIS (Fig. 1A and B). According to immunohistochemistry findings, breast specimens were scored for the expression of PKC- $\zeta$ by the pathologist (Fig. 1C). Normal tissue had no to moderate expression (score, 0,2), LCIS had abundant expression (scores, 3,3), ILC had moderate to strong expression (scores, 2,3+), DCIS had moderate to abundant expression (scores, 2.3), and IDC had abundant to strong expression (scores, $3,3+$ ). The most robust expression was found to be in ILC and IDC represented by the $3+$ score. However, ILC was found to have moderate to strong expression, whereas IDC was found to have abundant to strong expression. These findings suggest that $\mathrm{PKC}-\zeta$ is found higher in invading tissues when compared to non-invading tissues and more so in IDC when compared to ILC. These data suggest that the stage between in situ and invading malignancy can be correlated to an increased $\mathrm{PKC}-\zeta$ protein expression.

$P K C-\zeta$ and E-cadherin levels in tissue specimens. Since the decreased expression of E-cadherin is indicative of a more aggressive phenotype, the relationship between PKC- $\zeta$ and E-cadherin protein levels was studied. Western blot analysis was used in the context of probing for $\mathrm{PKC}-\zeta$ and E-cadherin in IDC breast tissues (Fig. 2A). We randomly selected 8 samples out of the 29 IDC tissue specimen to illustrate the data. We could not establish a significant relationship between PKC- $\zeta$ expression and E-cadherin expression in IDC samples. Our 
Table III. Chi-square statistical analysis results of normal and malignant breast subtypes (normal, LCIS, ILC, DCIS and IDC; $\mathrm{N}=83$ )

\begin{tabular}{lcccccccr}
\hline Variable & Normal & LCIS & ILC & DCIS & IDC & Row totals & Chi-square & P-value \\
\hline PKC- $\zeta$ present & 3 & 1 & 5 & 1 & 23 & 33 & a 32.6715 & $<0.00001$ \\
PKC- $\zeta$ absent & 29 & 2 & 8 & 5 & 6 & 50 & & \\
Column totals & 32 & 3 & 13 & 6 & 29 & 83 (grand total) & & \\
\hline
\end{tabular}

Values were considered to be statistically significant at $\mathrm{P}<0.01$. The Chi-square analysis compared the absence or presence of $\mathrm{PKC}-\zeta$ expression (determined by western blots) in different breast tissue specimens.LCIS, lobular carcinoma in situ; ILC, invasive lobular carcinoma; DCIS, ductal carcinoma in situ; IDC, invasive ductal carcinoma; PKC- $\zeta$, protein kinase C- $\zeta$.

Table IV. Chi-square statistical analysis results of estrogen receptor expression $(\mathrm{N}=25)$.

\begin{tabular}{lcccc}
\hline Variable & ER + & ER- & Row totals & Chi-square \\
\hline PKC- - present & 10 & 6 & 16 & 0.0434 \\
PKC- $\zeta$ absent & 6 & 3 & 9 & 0.834969 \\
Column totals & 16 & 9 & 25 (grand total) & \\
\hline
\end{tabular}

Values were considered to be not statistically significant at $\mathrm{P}<0.10$. The Chi-square analysis compared the absence and presence of PKC- $\zeta$ expression (determined by western blot) with the absence (-) and presence (+) of estrogen receptors (ER) in different breast tissue specimens. PKC- $\zeta$, protein kinase $C-\zeta$.

Table V. Chi-square statistical analysis results of Scarff-Bloom-Richardson grading $(\mathrm{N}=22)$.

\begin{tabular}{|c|c|c|c|c|c|c|}
\hline Variable & NG1 & NG2 & NG3 & Row totals & Chi-square & P-value \\
\hline PKC- $\zeta$ present & 4 & 4 & 5 & 13 & 0.2 & 0.90485 \\
\hline PKC- $\zeta$ absent & 3 & 2 & 4 & 9 & & \\
\hline Column totals & 7 & 6 & 9 & 22 (grand total) & & \\
\hline
\end{tabular}

Values were considered to be not statistically significant at $\mathrm{P}<0.01$. The Chi-square analysis compared the absence and presence of $\mathrm{PKC}-\zeta$ expression (determined by western blot) with nuclear grades 1-3 (NG1-NG3) in different breast tissue specimens. PKC- $\zeta$, protein kinase C- $\zeta$.

linear regression test $($ Fig. 2B) showed no significance $(\mathrm{P}<0.05)$ between the expressions of $\mathrm{PKC}-\zeta$ and $\mathrm{E}$-cadherin protein levels in 20 randomly selected IDC breast tissue samples. The $N$ value was too low to take into consideration for LCIS, ILC and DCIS. Normal breast tissues were not taken into consideration since the PKC- $\zeta$ expression was only found in 3 samples out of 32. However, our investigation established the relationship between E-cadherin protein expression and nuclear grade diagnosis (Fig. 2C). The Scarff-Bloom-Richardson scale data was derived from the pathology reports (summarized in Tables I and V). The results show that E-cadherin protein expression had an inverse relationship to nuclear grade diagnosis. Even though the PRKCZ gene is not one of the top mutated genes in breast cancer (carcinomas and carcinomas in situ), CDH1 is on the top of the list (Table VI) (3).

PRKCZ gene silencing decreases the invasion of breast cells. To investigate the effects of PKC- $\zeta$ inhibition on the invasive behavior of breast cancer cells, a Transwell invasion assay and immunostaining of F-actin were performed. When compared to the control, PKC- $\zeta$ knockdown decreased the invasion of breast cancer cells by $60 \%$ and was significant (Student's t-test $\mathrm{P}<0.05$, one-way ANOVA Tukey HSD P-value 0.0029646, $\mathrm{P}<0.01$; Scheffé $\mathrm{P}$-value 0.0049622, $\mathrm{P}<0.01$; Bonferroni P-value 0.0040067, $\mathrm{P}<0.01$ and Holm P-value 0.0040067, $\mathrm{P}<0.01$ ) (Fig. 3A and B). In addition, the levels of two important components of metastatic pathways, Racl and RhoA, were also decreased dramatically (Fig. 3C). Moreover, MDA-MB-231 breast cancer cells were fixed and stained with phalloidin probe to visualize the impacts of PRKCZ gene silencing on F-actin organization. The silencing of PRKCZ caused the reorganization of F-actin around the cell cytoskeleton (Fig. 4). Taken together, these data advance the theory that PKC- $\zeta$ modulates the invasive behavior of breast cancer cells by the regulation of invasion through the Racl/RhoA pathway and cytoskeleton filaments. 

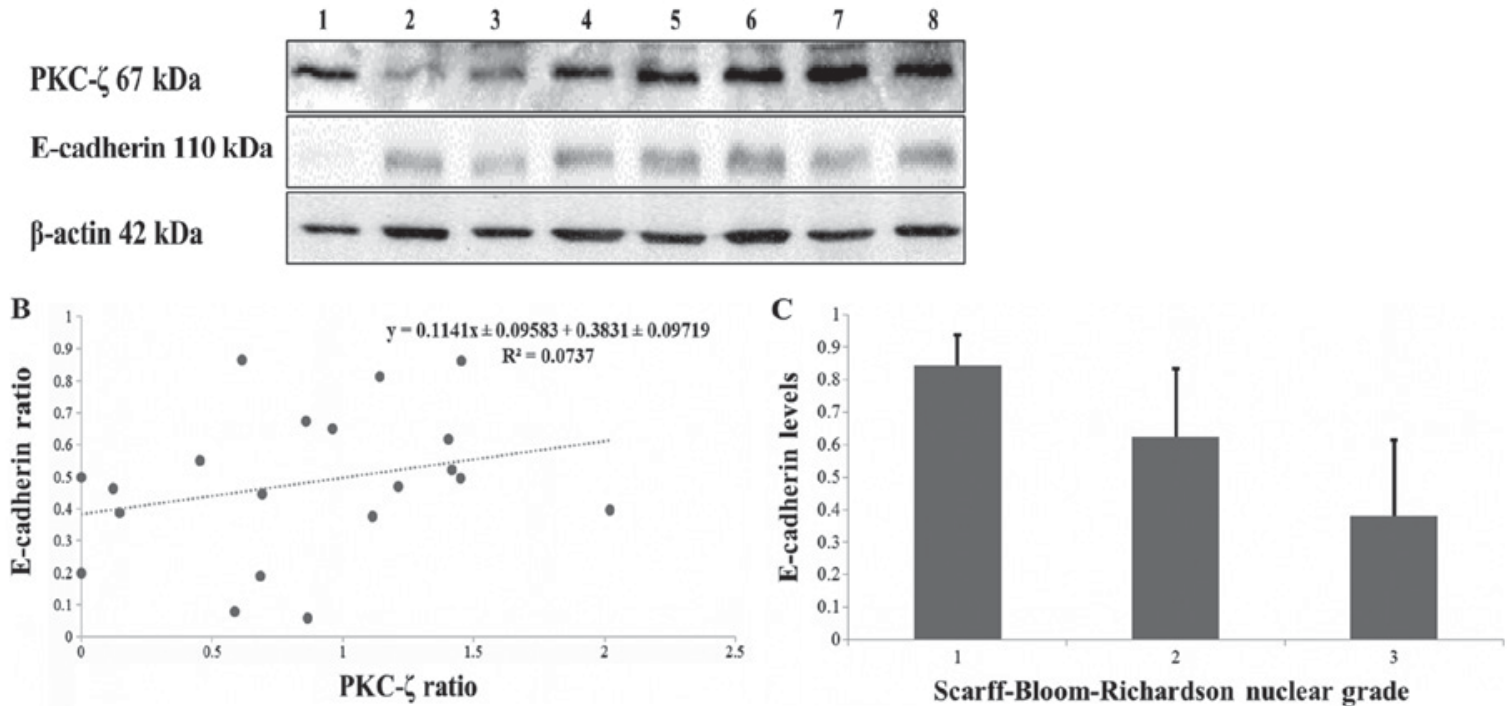

Figure 2. The non-linear relationship between E-cadherin expression and PKC- $\zeta$ expression and the E-cadherin ratio measured by tumor nuclear grade in IDC. (A) We investigated the expression of PKC- $\zeta$ in comparison to E-cadherin expression in IDC diagnosed breast tissues by western blot. The western blot represents 8 samples of IDC. (B) We normalized densities of PKC- $\zeta$ and E-cadherin protein expression based on $\beta$-actin levels and plotted them on a scattered plot graph to test for a linear relationship $(N=20)$. (C) The E-cadherin ratios were further compared to nuclear grade diagnosis listed on the pathology report, according to the Scarff-Bloom-Richardson scale. PKC- $\zeta$, protein kinase C- $\zeta$; IDC, invasive ductal carcinoma.
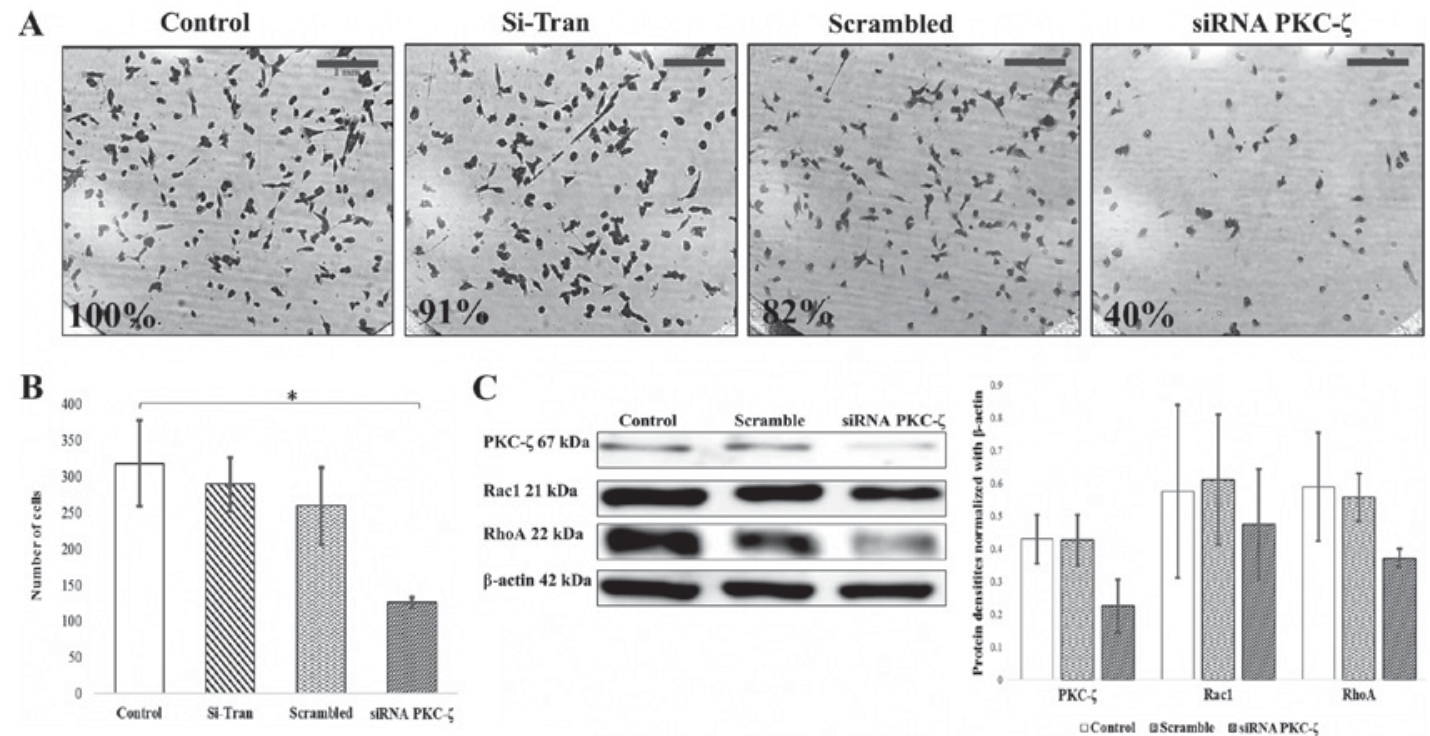

Figure 3. PKC- $\zeta$ knockdown retards invasion of MDA-MB-231 breast cancer cells. MDA-MB-231 breast cancer cells were placed in the upper chamber of Transwell plate coated with 0.5X BME and serum containing media was placed in the lower chamber as a chemoattractant. Following transfection with siPRKCZ and scrambled RNA, cells that invaded through the BME and migrated into the lower chamber were stained with crystal violet and (A) observed under a microscope at magnification, x10; scale bar, $1 \mathrm{~mm}$. Untreated cells and cells treated with the transfection reagent (Si-Tran) were also used to establish the effect on invasion. (B) The cells were counted for each treatment image $(N=3)$, averaged and analyzed with the standard deviation, a Student's t-test $(" \mathrm{P}<0.05)$ and a one-way ANOVA was also used to evaluate the data with the post-hoc Tukey's HSD test $(\mathrm{P}<0.01)$, Scheffé multiple comparison $(\mathrm{P}<0.01)$, Bonferroni $(\mathrm{P}<0.01)$ and Holm $(\mathrm{P}<0.01)$. All statistical tests were used to determine the statistical significance of the invasion data. $(\mathrm{C})$ The cells were further investigated by western blot to analyze the Rac1/RhoA pathway. This data was quantified and averaged for the graph with standard error of the mean represented. PKC- $\zeta$, protein kinase C- $\zeta$; BME, basement membrane extract; Rac1, Ras-related C3 botulinum toxin substrate 1; RhoA, Ras homolog gene family member A.

\section{Discussion}

Previously, Yin et al showed that the expression of PKC- $\zeta$ was higher in invading breast tissues compared to samples uncomplicated with tumors and the highest $\mathrm{PKC}-\zeta$ protein expression existed in stage III human breast ductal carcinomas (17). Likewise, Paul et al concluded that the depletion of PKC- $\zeta$ reduced the invasive behaviors of MDA-MB-231 cells by upregulating epithelial markers such as Zonula occludens-1 (ZO-1) and E-cadherin (6). They also found that PKC $-\zeta$ activation (phosphorylated PKC- $\zeta$ levels) was found higher in invasive tissues (i.e., IDC and metastatic tissues) when compared to non-invasive tissues (DCIS). PKC- $\zeta$ expression did not significantly change with the presence 
or absence of receptors (ER and HER2) (6). Our western blots and Chi-squared analysis (Fig. 1A and B; Table IV) support these findings. They performed a PKC- $\zeta$ knockdown mouse study and found that the depletion of PKC- $\zeta$ leads to an approximately 50\% reduction in primary tumor growth compared to the control within five weeks (6). Similarly, our western blot analysis and immunohistochemistry data of IDC and ILC (Fig. 1A-C) suggest that PKC- $\zeta$ might also be a novel component in pathways that affect cancer cell invasion and metastasis.

PKC- $\zeta$ assists a transcription factor $(\mathrm{NF \kappa B}-\mathrm{p} 65)$ that downregulates targets such as E-cadherin and ZO-1 (6). In addition, decreased E-cadherin levels cause the cells to no longer adhere to the extracellular matrix causing the cells to migrate, invade, or metastasize (6). Moreover, Chua et al showed that NF- $\mathrm{KB}$ induction elevated expression of Zinc finger E-box binding homeobox 1 and 2 (ZEB-1 and ZEB-2) which ultimately repressed the E-cadherin levels (7). In our investigation, we could not establish a statistical relationship between PKC- $\zeta$ and E-cadherin protein expression in IDC (Fig. 2B) as per the linear regression analysis. However, previous studies illustrated an increase in E-cadherin levels in PKC- $\zeta$ knockdown MDA-MB-231 cells (6).

In addition, our findings showed an increased expression of PKC- $\zeta$ in IDC tissues when compared to other subtypes (Fig. 1A-C) which may be because of the difference in pathological features of ductal and lobular tumors. Ductal carcinomas are lined with a two-layered stratified cuboidal epithelium resting on the basement membrane. This cuboidal epithelium contains tight junctions where E-cadherins are located and play a central role in cell-to-cell adhesion (18). In contrast, lobular carcinomas vary in terms of molecular and genetic aberrations. Lobular carcinomas are epithelial-like, growing individually in sheets or in a single file (4). In our investigation, E-cadherin levels were also compared to the nuclear grade listed on the pathology report (Fig. 2C; Table V), which supports the previous studies that described a decline in E-cadherin as a part of the epithelial to mesenchymal transition leads to metastasis $(7,19)$.

During metastasis, directional cell movement involves five distinct steps: leading edge membrane protrusion, adhesion of the protrusion to the substrates, cell body translocation, de-adhesion of the tail from the substrate and trailing edge retraction (20). These processes are mainly controlled by reorganization of the actin in the cell cytoskeleton which in turn are regulated by Guanosine Triphosphatases (GTPases) (21). Among the GTPases, RhoA, Rac1, and CDC42 are most commonly studied because of their crucial role in actin assembly and formation of metastatic structures of cells, such as filopodia, lamellipodia and stress fibers (22). Rac1 and CDC42 produce localized actin polymerization at the leading edge which pushes the membrane forward in slender like structure known as filopodia and sheet-like structure known as lamellipodia that ultimately generate locomotive force in migrating cells (23). In contrast, RhoA promotes the assembly of contractile actomyosin filaments and acts on the rear end of the migrating cells, inducing tail detachment (24). Thus, Rac1 and CDC42 stimulate leading edge protrusion, and RhoA stimulates trailing edge retraction in metastatic cells. We found that the knockdown of PKC- $\zeta$ by siPRKCZ

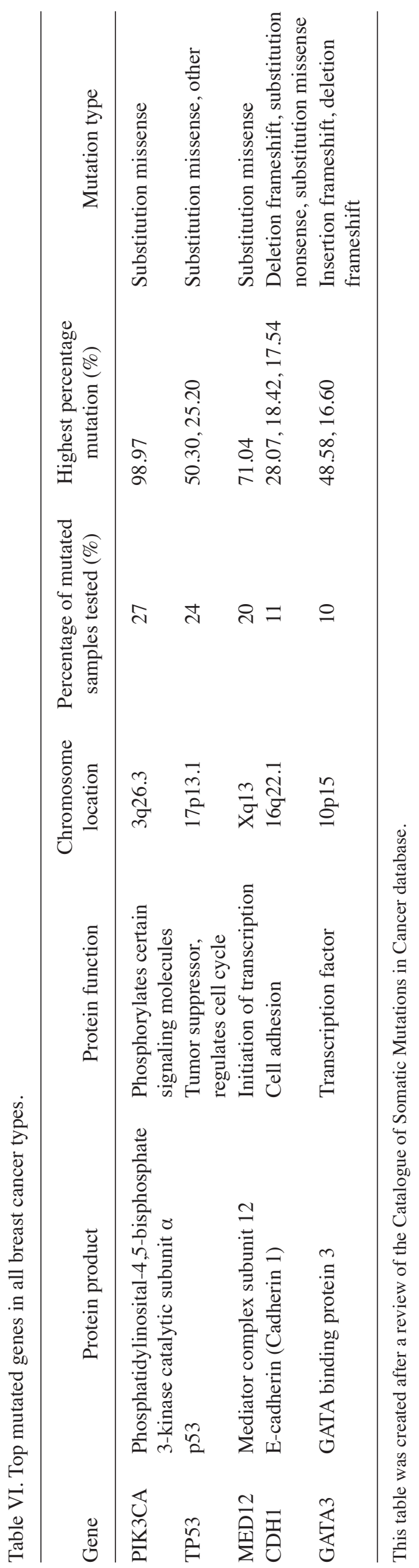



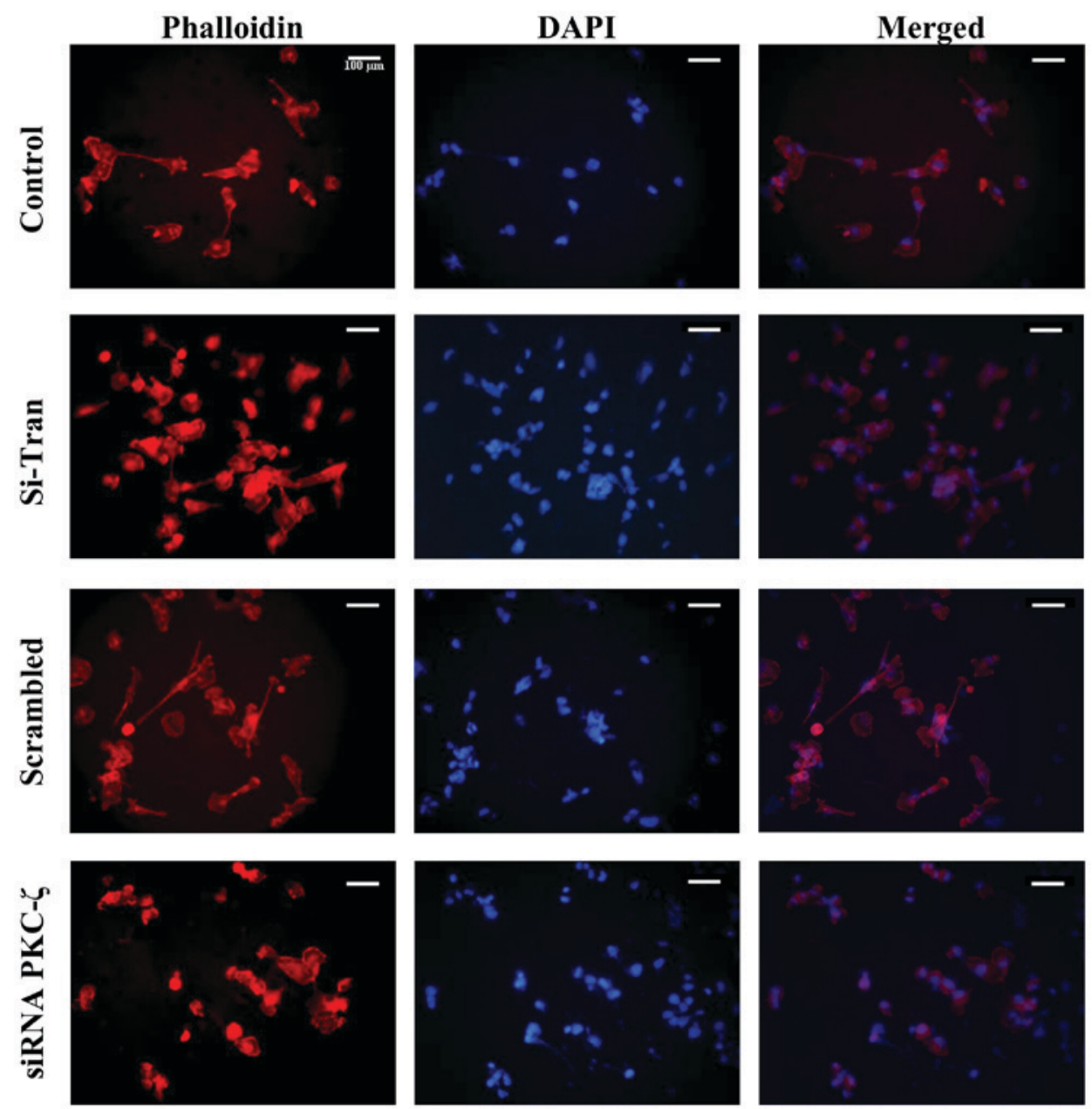

Figure 4. Organization of F-actin upon the knockdown of PKC- $\zeta$. MDA-MB-231 breast cancer cells were treated with SiTran, scrambled RNA and siPRKC- $\zeta$ for $24 \mathrm{~h}$. The cells that received no treatment were used as the control. These cells were then stained with 1X Phalloidin-iFluor 594 and counterstained with DAPI. Scale bar, $100 \mu \mathrm{m}$. Original magnification, x20. DAPI, 4',6-diamidino-2-phenylindole; F-actin, filamentous actin; PKC- $\zeta$, protein kinase C- $\zeta$.

reduced the invasion of MDA-MB-231 breast cancer cells by $60 \%(\mathrm{P}<0.05)$ when compared to control (Fig. 3A and B). In addition, there was a decreased expression of both Racl and RhoA in siPRKCZ transfected MDA-MB-231 breast cancer cells compared to control (Fig. 3C). Furthermore, our immunostaining analysis of F-actin illustrated that actin filaments were nicely organized around the cells with the inhibition of PKC- $\zeta$ (Fig. 4). Hence, PKC- $\zeta$ may play an essential role in the invasion and migration of breast cancer cells by the regulation of RhoA and Rac1 pathways.

To conclude, our findings suggest that PKC- $\zeta$ is found to be most abundant in invading tissue subtypes and may be a functional component of invasion pathways such as Rac1 and RhoA. The use of PKC- $\zeta$-specific inhibitors could be used to correlate the decrease in expression or functionality of PKC- $\zeta$ with the decrease in invasive behavior in breast cancer.

\section{Acknowledgements}

The authors would like to thank the Tissue Core, Moffitt Cancer Center (Tampa, FL, USA) for their assistance. Tissue samples were provided by the NCI Cooperative Human Tissue Network.

\section{Funding}

This study was funded by the University of South Florida (Tampa, FL, USA) Foundations 42-0142. The authors would like to acknowledge the financial contributions from the William and Ella Owens Medical Research Foundation, Alaska Run for Women, Save the Ta-Tas Foundation, Mary Ewell Dickens Foundation, Yolanda and Salvatore Gigante Charitable Foundation Trust, Daniel Tanner Foundation, Frederick H. Leonhardt Foundation and the Charles and Ann Johnson Foundation.

\section{Availability of data and materials}

The datasets we used and/or analyzed during the current study are available from the corresponding authors upon reasonable request. The datasets we generated and/or analyzed during the current study are available from the COSMIC repository at https://cancer.sanger.ac.uk/cosmic.

\section{Authors' contributions}

TS contributions include tissue selection and fractionation, western blot analysis, densitometry analysis, statistical analyses, 
pathway analysis and writing. SMAI contributions include the invasion assay, phalloidin staining, and writing. CA contributions include preliminary western blot analysis. AA contributions to the paper include statistical analysis of E-cadherin expression and nuclear grade. MAD contributions to the paper include concept, design, writing, editing, resources, supervision and funding acquisition. The authors read and approved the final manuscript.

\section{Ethics approval and consent to participate}

The right to informed consent was waived by the Ethics committee of the University of South Florida.

\section{Patient consent for publication}

Not applicable.

\section{Competing interests}

The authors declare that they have no competing interests.

\section{Author information}

TS is currently a graduate student at the University of South Florida, Department of Chemistry. She is working on the second year of her PhD and continues to work on ovarian cancer and breast cancer.

\section{References}

1. Siegel RL, Miller KD and Jemal A: Cancer statistics, 2016. CA Cancer J Clin 66: 7-30, 2016.

2. Kuhn E: Quick Guide for Most Commonly Used Breast Cancer Statements. Susan G. Komen: 10-11, 2015.

3. COSMIC: Catalogue of somatic mutations in cancer, 2016.

4. Ruibal A, Núñez MI, del Rio M, Arias J, Martínez MI, Rabadán J and Tejerina A: Clinical-biological differences between invasive ductal carcinomas and breast lobular carcinomas. Preliminary results. Rev Esp Med Nucl 18: 84-87, 1999.

5. Hoesel B and Schmid JA: The complexity of NF- $\kappa$ B signaling in inflammation and cancer. Mol Cancer 12: 86, 2013.

6. Paul A, Danley M, Saha B, Tawfik O and Paul S: PKC $\zeta$ promotes breast cancer invasion by regulating expression of E-Cadherin and Zonula Occludens-1 (ZO-1) via NFKB-p65. Sci Rep 5: 12520, 2015.

7. Chua HL, Bhat-Nakshatri P, Clare SE, Morimiya A, Badve S and Nakshatri H: NF-kappaB represses E-cadherin expression and enhances epithelial to mesenchymal transition of mammary epithelial cells: Potential involvement of ZEB-1 and ZEB-2. Oncogene 26: 711-724, 2007.
8. Singhai R, Patil VW, Jaiswal SR, Patil SD, Tayade MB and Patil AV: E-Cadherin as a diagnostic biomarker in breast cancer. N Am J Med Sci 3: 227-233, 2011.

9. Hirai T and Chida K: Protein kinase Czeta (PKCzeta): Activation mechanisms and cellular functions. J Biochem 133: 1-7, 2003.

10. Islam SMA, Patel R and Acevedo-Duncan M: Protein Kinase $\mathrm{C}-\zeta$ stimulates colorectal cancer cell carcinogenesis via PKC- $\zeta /$ Rac1/Pak $1 / \beta-$ Catenin signaling cascade. Biochim Biophys Acta Mol Cell Res 1865: 650-664, 2018.

11. Wu J, Liu S, Fan Z, Zhang L, Tian Y and Yang R: A novel and selective inhibitor of PKC $\zeta$ potently inhibits human breast cancer metastasis in vitro and in mice. Tumour Biol 83: 8391-8401, 2016.

12. Lin YM, Su CC, Su WW, Hwang JM, Hsu HH, Tsai CH, Wang YC, Tsai FJ, Huang CY, Liu JY and Chen LM: Expression of protein kinase $\mathrm{C}$ isoforms in cancerous breast tissue and adjacent normal breast tissue. Chin J Physiol 55: 55-61, 2012.

13. Schöndorf T, Kurbacher CM, Becker M, Warm M, Kolhagen H and Göhring UJ: Heterogeneity of proteinkinase $\mathrm{C}$ activity and PKC-zeta expression in clinical breast carcinomas. Clin Exp Med 1: 1-8, 2001.

14. Kim SW, Roh J and Park CS: Immunohistochemistry for pathologists: Protocols, pitfalls, and Tips. J Pathol Transl Med 50: 411-418, 2016.

15. GraphPad Software: GraphPad QuickCalcs: Linear regression calculator. 2018.

16. Social science statistics: Chi-square test calculator-up to $5 \times 5$ contingency table. 2018

17. Yin WJ, Lu JS, Di GH, Lin YP, Zhou LH, Liu GY, Wu J, Shen KW, Han QX, Shen ZZ and Shao ZM: Clinicopathological features of the triple-negative tumors in Chinese breast cancer patients. Breast Cancer Res Treat 115: 325-333, 2009.

18. Hugo HJ, Gunasinghe NPAD, Hollier BG, Tanaka T, Blick T, Toh A, Hill P, Gilles C, Waltham M and Thompson EW: Epithelial requirement for in vitro proliferation and xenograft growth and metastasis of MDA-MB-468 human breast cancer cells: Oncogenic rather than tumor-suppressive role of E-cadherin. Breast Cancer Res 19: 86, 2017.

19. Onder TT, Gupta PB, Mani SA, Yang J, Lander ES and Weinberg RA: Loss of E-cadherin promotes metastasis via multiple downstream transcriptional pathways. Cancer Res 68: 3645-3654, 2008.

20. Lauffenburger DA: Cell motility. Making connections count. Nature 383: 390-391, 1996.

21. Raftopoulou M and Hall A: Cell migration: Rho GTPases lead the way. Dev Biol 265: 23-32, 2004.

22. Lawson CD and Ridley AJ: Rho GTPase signaling complexes in cell migration and invasion. J Cell Biol 217: 447-457, 2018.

23. Small JV, Stradal T, Vignal E and Rottner K: The lamellipodium: Where motility begins. Trends Cell Biol 12: 112-120, 2002.

24. Nobes CD and Hall A: Rho GTPases control polarity, protrusion, and adhesion during cell movement. J Cell Biol 144: 1235-1244, 1999. International (CC BY-NC-ND 4.0) License. 